\title{
Relação entre o número de consultas do pré-natal e desfechos adversos perinatais em pacientes de baixo risco
}

\section{Relation between the number of prenatal consultations and adverse perinatal outputs in patients with low risk}

DOI: $10.46919 / \operatorname{archv2n5-006~}$

Recebimento dos originais: 01/05/2021

Aceitação para publicação: 31/06/2021

\section{João Pedro Ribeiro Baptista}

Estudantes de Medicina na Universidade da Região de Joinville - UNIVILLE - Joinville (SC), Brasil.

\section{Guilherme Schroder Stepic}

Estudantes de Medicina na Universidade da Região de Joinville - UNIVILLE - Joinville (SC), Brasil.

\section{Júlia Opolski Nunes da Silva}

Estudantes de Medicina na Universidade da Região de Joinville - UNIVILLE - Joinville (SC), Brasil.

\section{Rodrigo Ribeiro e Silva}

Estudantes de Medicina na Universidade da Região de Joinville - UNIVILLE - Joinville (SC), Brasil.

\section{Dieter Alisson Neumann}

Estudantes de Medicina na Universidade da Região de Joinville - UNIVILLE - Joinville (SC), Brasil.

\section{Iramar Baptistella do Nascimento}

Departamento de Ciência em Saúde - Uniersidade de Santa Catarina - CEFID - Florianópolis (SC), Brasil

\section{Carla Gisele Vaichulonis}

Enfermeira na Maternidade Darcy Vargas - Coordenadora do Centro de Estudos - Joinville (SC), Brasil.

\section{Jean Carl Silva}

PhD em Ciências Médicas (UNIFESP/SP); Supervisor do Setor de Alto Risco da Maternidade Darcy Vargas; Professor Adjunto na Universidade da Região de Joinville - UNIVILLE - Joinville (SC), Brasil.

\section{RESUMO}

Objetivo: Avaliar os impactos do número de consultas realizadas no acompanhamento de pré-natal na rede básica sobre os desfechos adversos. Metodologia: Estudo de corte transversal, foram selecionadas aleatoriamente puérperas de risco habitual com mais de 18 anos que fizeram pré-natal na rede pública de Joinville-SC, com gestação única. Os desfechos avaliados foram prematuridade, número de cesarianas, internação de UTI neonatal e baixo peso ao nascer. O cálculo da razão de chance teve intervalo de confiança de 95\%. Resultados: Comparamos as características e desfechos materno-fetais de pacientes que fizeram 5 consultas ou menos $(n=109)$, puérperas com 6 ou 7 consultas $(n=146)$ e pacientes que realizaram $8(n=430)$. Dos resultados do perfil materno, foram significativos, os resultados socioeconômicos como idade, gestações anteriores, escolaridade, raça, estado civil, profissão, número de pessoas na casa e remuneração. Do recém-nascido encontramos diferenças no capurro e peso, ambos maiores em pacientes com mais de 8 consultas. No cálculo da razão de chance ajustado encontramos redução na prematuridade nas pacientes que fizeram 6 ou 7 consultas $(0,26$ IC95\% 0,073-0,928) e nas que realizaram 8 ou mais $(0,06$ IC95\% 0,016- 
0,229). Não encontramos diferença no número de cesarianas, baixo peso ao nascer e na internação de UTI neonatal em nenhuma das populações. Conclusão: A realização de 6 ou 7 consultas de pré-natal reduz 74\% as chances de prematuridade, enquanto a realização de 8 ou mais consultas diminuiu em $94 \%$.

Palavras-chave: Complicações na Gravidez. Cuidado Pré-natal. Recém-Nascido Prematuro.

\begin{abstract}
Objetive: Evaluate the impacts of the number of prenatal consultations during medical follow-up in the basic health care system over adverse outcomes. Methodology: Cross-sectional cohort study, selected randomly puerperal women with habitual risk with at least 18 year old who did prenatal care in the public health care system in Joinville-SC, with single pregnancy. The adverse outcomes evaluated were prematurity, number of caesarean sections, neonatal ICU admission and low birth weight. The odds ratio calculation had a confidence interval of $95 \%$. Results: We compared characteristics and maternal-fetal outcomes of patients with 5 consultations or less $(n=109)$, puerperal women with 6 or 7 consultations $(n=146)$ and patients with $8(n=430)$. Of the maternal profile results found, were significant, the socioeconomic results like age, number of pregnancy, schooling, race, marital status, profession, number of people living in the house and salary. Of the newborns, we found differences in the capurro and weight; both were higher in patients with more than 8 consultations. In the adjusted odds ration calculation we found reduction in prematurity who did 6 or 7 consultations $(0,26 \mathrm{CI} 95 \% 0,073-0,928)$ and in the 8 or more consultations group $(0,06$ CI95\% 0,016-0,229). We did not found difference in the number of caesarean sections, low birth weight and neonatal ICU admissions in any of the populations. Conclusion: The realization of 6 or 7 prenatal consultations reduces in $74 \%$ the chances of prematurity, while the realization of 8 or more consultations reduces it in $94 \%$.
\end{abstract}

Keywords: Pregnancy Complications. Prenatal Care. Infant, Premature.

\title{
1 INTRODUÇÃO
}

A atenção pré-natal tem como objetivos principais o rastreio de possíveis situações de risco, bem como promover a saúde do binômio mãe-feto e auxiliar no tratamento rápido e eficiente de intercorrências que possam ocorrer durante a gestação. Ela é de fundamental importância e imprescindível para reduzir a mortalidade materna e perinatal. (1-2)

A atenção pré-natal tem como objetivos principais o rastreio de possíveis situações de risco, bem como promover a saúde do binômio mãe-feto e auxiliar no tratamento rápido e eficiente de intercorrências que possam ocorrer durante a gestação. O acompanhamento da gestação é de fundamental importância e imprescindível para reduzir a mortalidade materna e perinatal (2).

O Ministério da saúde do Brasil classifica como acompanhamento ideal aquele que apresentar um número mínimo de 6 consultas (3), sendo essas divididas em uma no primeiro, duas no segundo e três no terceiro trimestre de gestação. Entretanto, em 2016 esse protocolo do governo ficou desatualizado, pois a Organização Mundial da Saúde pública uma nova diretriz para a atenção primária colocando como o correto ser o número mínimo de 8 consultas (4). 
A literatura ilustra a relação de um maior número de consultas com os desfechos adversos maternofetais como um fator de proteção em especial para a prematuridade (5). E tendo em vista a prematuridade como principal desfecho neonatal as consultas pré-natal diminuem a sua incidência, elas também ao longo do período gestacional facilitam o tratamento e controle de pré-eclâmpsia, Diabetes Mellitus Gestacional, entre outros (6).

Em vista do exposto, é interessante avaliar o número de consultas pré-natal da gestante de baixo risco na Rede Básica, relacionando com os possíveis desfechos adversos materno-fetais ocorridos, levantando hipóteses a partir dos resultados comparando eles aos padrões da OMS e do Ministério da Saúde.

\section{METODOLOGIA:}

Trata-se de um estudo corte transversal. Foi realizada a aplicação de um questionário que contemplou aspectos de todo processo do Pré-Natal, em uma amostra aleatória estratificada de puérperas, correspondente a $10 \%$ da população atendida na Maternidade Darcy Vargas (MDV). A amostra foi selecionada aleatoriamente através do programa "Randomized Research" através da indicação de 10 dias por mês no qual os pesquisadores deveriam aplicar as entrevistas a todas as puérperas que tiveram seus partos na Maternidade Darcy Vargas. A população foi dividida em 3 grupos, pacientes que fizeram 5 consultas ou menos durante o pré-natal, pacientes que realizaram 6 ou 7 consultas, dentro da faixa recomendada pelo Ministério da Saúde, e pacientes que fizeram 8 consultas ou mais, conforme o recomendado pela OMS.

A coleta de dados teve início após a autorização do Comitê de Ética em Pesquisa. O projeto foi aprovado sob o número CAAE 82477318.1.0000.5363 pelo Comitê de Ética em Pesquisa (CEP) do Hospital Regional Hans Dieter Schmidt, Joinville, SC, Brasil. O estudo seguiu os critérios definidos pela Resolução 466/2012 cada puérpera assinou o Termo de Consentimento Livre e Esclarecido presencialmente.

18 anos com gestação única que falam português e realizaram o pré-natal em Unidades de Saúde do Sistema Único de Saúde da cidade de Joinville - SC cujo parto tenha ocorrido na Maternidade Darcy Vargas (MDV) e que voluntariamente queiram participar desta pesquisa, mediante assinatura Termo de Consentimento Livre e Esclarecido. Foi informado o livre arbítrio de deixar de participar da pesquisa a qualquer momento da entrevista. $\mathrm{O}$ critério de exclusão de pacientes foi: puérperas que se recusaram a participar da pesquisa após o início do questionário.

Analisou-se os seguintes dados da população a fim de compreender seu perfil: características socioeconômicas da puérpera (idade, raça, escolaridade, renda, profissão remunerada, estado civil), uso de substâncias (álcool, cigarro e drogas) durante a gravidez, antecedentes obstétricos, complicações na gestação (DHEG, DMG e ITU), planejamento, perfil do pré-natal (número de consultas, IG da primeira 
consulta), características do recém-nascidos e desfechos adversos perinatais até 48 horas após o parto. Todos os dados foram obtidos através de entrevista com escuta qualificada, consulta a Caderneta da Gestante, ao Plano de Parto e ao Prontuário Eletrônico.

Concomitantemente à coleta foi realizada a digitalização dos dados em um banco eletrônico com dupla entrada, para verificação de concordância e possíveis erros de digitação. Foi utilizado o software estatístico Statistical Package for the Social Sciences (SPSS), versão 21.0, para análise estatísticas dos dados. Todas as variáveis foram analisadas descritivamente, assim, as variáveis contínuas (numérica) foram estudadas por meio do cálculo de médias e desvios-padrão. Para as variáveis qualitativas calcularam-se frequências absolutas e relativas. Para a verificação da hipótese de igualdade entre as médias dos grupos, foi utilizado teste $\mathrm{T}$ de student, quando a distribuição foi normal, e o teste não paramétrico de Kruskal wallis, quando o teste de normalidade foi recusado, tendo em vista a existência de 3 grupos distintos. O teste de normalidade utilizado foi o Kolmogorov-Smirnov. Para se provar a homogeneidade dos grupos em relação às proporções, utilizou-se o teste Qui-quadrado ou o teste exato de Fisher para frequências abaixo de 5 .

Modelos de regressão logística multinomial foram construídos de modo a analisar a influência do número de consultas pré-natal sobre os desfechos estudados (internação em UTI neonatal, prematuridade e baixo peso ao nascer). Fatores de confusão foram: idade materna, primigestas, DHEG, DMG, ITU, uso de álcool e tabaco na gestação. Desse modo, estimou-se a relevância do efeito das variáveis pelo cálculo da razão de chances (Odds Ratio - OR) ajustada conforme fatores de confusão, com seus respectivos intervalos de confiança de $95 \%$ (IC95\%). Os valores foram considerados significativos quando $P<0,05$.

\section{RESULTADOS}

Devido à forte relação entre número de consultas e melhor qualidade gestacional encontrada em alguns estudos, foram comparadas as características e desfechos materno-fetais de pacientes que fizeram 5 consultas ou menos $(n=109 / 15,9 \%)$, puérperas com 6 ou 7 consultas $(n=146 / 21,3 \%)$ e pacientes que realizaram $8(n=430 / 62,8 \%)$.

Em relação ao perfil socioeconômico das gestantes, para fins de análise foram significativos os resultados coletados nos quesitos de idade, gestação, raça, escolaridade, estado civil, profissão e número de pessoas que residem na casa. Em contrapartida não houve significância estatística os índices de renda familiar, fumo, álcool, Diabetes Mellitus Gestacional, Infecção do Trato Urinário, Doença Hipertensiva Específica da Gestação.

Quanto aos resultados do recém-nascido, foram encontradas diferenças significativas no Escore de Capurro, demostrando que ocorreu uma relação de maior escore com maior número de consultas. Além do peso do recém-nato aumentar também conforme o aumento do número de consultas. Outro dado relevante 
foi a prematuridade que se apresentava mais incidente em gestantes com 5 consultas ou menos, entretanto as classificações de moderadamente, muito e prematuro extremo não tiveram significância estatística.

No cálculo da razão de chance foi vista redução na prematuridade nas pacientes que fizeram 6 ou 7 consultas $(0,26$ IC95\% 0,073-0,928) e nas que realizaram 8 ou mais $(0,06$ IC95\% 0,016-0,229). Não se encontrou diferença no número de cesarianas, baixo peso ao nascer e na internação de UTI neonatal em nenhuma das populações.

Tabela 1 - Características maternas relacionadas ao número de consultas: *

\begin{tabular}{|c|c|c|c|c|}
\hline & $\begin{array}{l}\leq 5 \text { consultas } \\
(n=109)\end{array}$ & $\begin{array}{l}6-7 \text { consultas } \\
(n=146)\end{array}$ & $\begin{array}{l}\geq 8 \text { consultas } \\
(n=430)\end{array}$ & $P$ \\
\hline IDADE & $25,44(6,4)$ & $27,76(5,9)$ & $27,85(6,2)$ & $0,015 * *$ \\
\hline GESTA & $2,89(1,3)$ & $3,12(1,7)$ & $2,24(1,2)$ & $0,000 * *$ \\
\hline $\mathrm{N}^{\circ}$ DE CONSULTA & $3,81(1,3)$ & $6,58(0,5)$ & $9,30(1,3)$ & $0,000 * *$ \\
\hline $\mathbf{R A C ̧ A ~}$ & & & & $0,015 * * *$ \\
\hline BRANCA & $59(54,1)$ & $91(62,3)$ & $309(66,2)$ & \\
\hline NEGRA & $11(10,1)$ & $15(10,3)$ & $41(8,8)$ & \\
\hline PARDA & $38(34,9)$ & $35(24,0)$ & $100(21,4)$ & \\
\hline OUTRAS & $1(7,11)$ & $5(3,5)$ & $17(3,6)$ & \\
\hline ESCOLARIDADE & & & & $0,002 * * *$ \\
\hline PRIMÁRIO & $45(41,3)$ & $40(27,4)$ & $102(21,8)$ & \\
\hline SECUNDÁRIO & $55(50,5)$ & $87(59,6)$ & $295(63,2)$ & \\
\hline SUPERIOR & $9(8,3)$ & $19(13,0)$ & $70(15,0)$ & \\
\hline ESTADO CIVIL & & & & $0,019 * * *$ \\
\hline SOLTEIRA & $41(37,6)$ & $56(38,4)$ & $120(25,7)$ & \\
\hline CASADA & $27(24,8)$ & $44(30,1)$ & $194(41,5)$ & \\
\hline UNIÃO ESTÁVEL & $37(33,9)$ & $40(27,4)$ & $138(29,6)$ & \\
\hline
\end{tabular}




\begin{tabular}{|c|c|c|c|c|}
\hline OUTRAS & $4(3,7)$ & $6(4,1)$ & $15(3,2)$ & \\
\hline PROFISSÃO & & & & $0,029 * * *$ \\
\hline REMUNERADA & $50(45,9)$ & $73(50,0)$ & $272(54,7)$ & \\
\hline NÃO REMUNERADA & $59(54,1)$ & $73(50,0)$ & $195(41,8)$ & \\
\hline $\begin{array}{l}N^{\circ} \text { DE PESSOAS NA } \\
\text { CASA }\end{array}$ & $4,63(1,3)$ & $4,36(1,4)$ & $3,97(1,2)$ & $0,002 * * *$ \\
\hline
\end{tabular}

\section{RENDA FAMILIAR}

\begin{tabular}{|c|c|c|c|c|}
\hline SEM & $1(0,9)$ & $2(1,4)$ & $5(1,1)$ & \\
\hline $1 \mathrm{SAL}$ & $8(7,3)$ & $15(10,3)$ & $22(4,7)$ & \\
\hline 1-2 SAL & $42(38,5)$ & $44(30,1)$ & $117(25,1)$ & \\
\hline $2-5 \mathrm{SAL}$ & $44(40,4)$ & $69(47,3)$ & $259(55,5)$ & \\
\hline$>5 \mathrm{SAL}$ & $9(8,3)$ & $8(5,5)$ & $44(9,4)$ & \\
\hline NI & $5(4,6)$ & $8(5,5)$ & $20(4,3)$ & \\
\hline FUMO & $10(9,2)$ & $9(6,2)$ & $19(4,1)$ & $0,085 * * *$ \\
\hline ÁLCOOL & $4(3,7)$ & $8(5,5)$ & $26(5,6)$ & $0,721 * * * *$ \\
\hline DHEG & $11(10,1)$ & $14(9,6)$ & $48(10,3)$ & $0,971 * * *$ \\
\hline DMG & $7(6,4)$ & $5(3,4)$ & $18(3,9)$ & $0,426 * * *$ \\
\hline ITU & $39(35,8)$ & $54(37,0)$ & $166(35,5)$ & $0,951 * * *$ \\
\hline
\end{tabular}

*Média e desvio-padrão, números absolutos e percentagens; **Kruskal wallis test; *** Teste Qui-quadrado; ****Teste Exato de Fisher; IG - Idade Gestacional; DMG - Diabetes Mellitus Gestacional; DHEG - Doença Hipertensiva Especifica da Gestação; ITU- Infecção do Trato Urinário.

Tabela 2 - Características do recém-nascido relacionadas ao número de consultas:

\begin{tabular}{lllll}
\hline & 5 ou $>$ consultas & 6 a 7 consultas & 8 ou < consultas & $P$ \\
\hline CAPURRO & $38,15(1,7)$ & $38,76(1,8)$ & $39,24(1,1)$ & 0,000 \\
PESO & $3100,56(430,9)$ & $3107,42(524,4)$ & $3340,47(428,6)$ & 0,000
\end{tabular}




\begin{tabular}{lllll} 
APGAR 1 $^{\circ}$ & $7,78(1,3)$ & $7,55(1,3)$ & $7,74(1,2)$ & 0,656 \\
\hline APGAR 5 $^{\circ}$ & $8,93(0,4)$ & $8,91(0,4)$ & $8,88(0,8)$ & 0,207 \\
\hline CESARIANA & $34(33,7)$ & $40(30,3)$ & $149(35,2)$ & 0,579 \\
\hline APGAR BAIXO 1 & $10(9,2)$ & $11(7,5)$ & $26(5,6)$ & 0,332 \\
\hline PREMATURIDADE & $18(16,5)$ & $10(6,8)$ & $8(1,7)$ & 0,000 \\
\hline MODERADO PREMATURO & $13(72,2)$ & $7(70)$ & $8(100)$ & 0,228 \\
\hline MUITO PREMATURO & $1(5,6)$ & $3(30)$ & 0 & 0,075 \\
\hline EXTREMO PREMATURO & $4(22,2)$ & 0 & 0 & 0,105 \\
\hline UTI NEO & $8(7,3)$ & $7(4,8)$ & $11(3,6)$ & 0,029 \\
\hline BAIXO PESO & $13(11,9)$ & $9(6,2)$ & $11(2,4)$ & 0,000 \\
\hline
\end{tabular}

*Média e desvio-padrão, números absolutos e percentagens; **Kruskal wallis test; *** Teste Qui-quadrado; ****Teste Exato de Fisher.

Tabela 3 - Desfechos Adversos

\begin{tabular}{|c|c|c|c|c|c|}
\hline & & & $P$ & $\mathrm{RC}$ & IC $95 \%$ \\
\hline $\begin{array}{l}\text { DESFECHOS ADVERSOS } \\
\text { CONSULTAS: }\end{array}$ & EM & $\geq 8$ & & & \\
\hline PREMATURIDADE & & & 0,000 & 0,060 & $0,016-0,229$ \\
\hline UTI NEO & & & 0,459 & 1,719 & $0,409-7,222$ \\
\hline BAIXO PESO & & & 0,808 & 0,846 & $0,220-3,252$ \\
\hline $\begin{array}{l}\text { DESFECHOS ADVERSOS } \\
\text { CONSULTAS: }\end{array}$ & EM & $6-7$ & & & \\
\hline PREMATURIDADE & & & 0,038 & 0,260 & $0,073-0,928$ \\
\hline UTI NEO & & & 0,405 & 1,836 & $0,439-7,679$ \\
\hline BAIXO PESO & & & 0,963 & 0,968 & $0,243-3,853$ \\
\hline
\end{tabular}

Fatores de confusão: idade materna, primigestas, DHEG, DMG, ITU, uso de álcool e tabaco na gestação.

\section{DISCUSSÃO}

Tendo em vista a forte associação entre o número de consultas no acompanhamento pré-natal com desfechos adversos materno-fetais encontrado na literatura (5). Foram confrontadas as características e os desfechos em mães que fizeram 5 consultas ou menos ( $\mathrm{n}=109$ / 15,9\%), com as pacientes que efetuaram 6 ou 7 consultas ( $n=146 / 21,3 \%$ ) e com as que realizaram 8 ou mais consultas ( $n=430 / 62,8 \%$ ). No cálculo 
de razão de chance, percebeu-se que a realização de 8 consultas ou mais reduziu em $94 \%$ os casos de prematuridade, já a realização de 6 a 7 consultas diminuiu em 74\%, quando comparadas a pacientes com menos de 5 consultas.

Do exposto é perceptível que mais da metade das gestantes estão adequadas às recomendações da OMS (4), realizando 8 ou mais consultas pré-natal. Isso ilustra um dado significativamente positivo por resultar em menor chance de desfechos adversos materno-fetais. Outro dado relevante é que menos de $16 \%$ do número de pacientes realizou 5 ou menos consultas, ou seja, mais de $80 \%$ da população está adequada às diretrizes governamentais (3) relacionadas à saúde gestacional. Esses dados indicam um bom desempenho, ainda mais quando comparado com outras localidades do Brasil. A realidade hoje no centrosul do país é de entre 70 e $80 \%$ das gestantes terem, no mínimo, 6 consultas pré-natal. Já a cidade de Joinville apresentou uma porcentagem acima disso, avaliando com, no mínimo, 8 encontros pré-natal. (7)

Em relação ao perfil das gestantes, no estudo foram significativos os resultados socioeconômicos como idade, gestações anteriores, escolaridade, raça, estado civil, profissão, número de pessoas na casa e remuneração. Esses dados ilustraram que mães solteiras, de raça parda ou negra, com menor escolaridade, não remuneradas e com mais gestações tiveram maior chance de não realizarem o acompanhamento prénatal adequado. Esse achado condiz com a literatura que também traça esse mesmo perfil epidemiológico materno que não comparece a consultas ao longo da gestação. Gestantes com acompanhamento da gravidez não condizente com a prerrogativa do SUS tem maior perspectiva de ser solteira. Porém, os resultados obtidos daqueles estudos revelam uma disparidade percentual mais expressiva que deste artigo. Isso ocorre devido a diferenças metodológicas no que tange a entrevista aplicada, naqueles abordando somente se é casada ou não, preterindo o quesito "união estável" que é abordada nesse artigo. $(8,9)$

Já sobre o perfil do recém-nascido, foi significativo os resultados, os quais ilustraram que neonatos que tiveram mais consultas, apresentaram melhor Capurro, maior peso, com menor porcentagem de Baixo Peso ao Nascer, UTI neonatal e prematuridade. Esse perfil segue o que é encontrado na literatura, que também ilustra que um neonato de gestante com mais consultas pré-natal é mais pesado, tem um Capurro mais adequado (10).

O resultado verificado na tabela sobre prematuridade, revelou que esta caía à medida que aumentava o número de consultas. Gestantes com mais de 8 consultas obtiveram menor índice de prematuridade que as com menos de 5 consultas. Isso ocorre por um maior cuidado na atenção primária à mãe, sendo que as consultas que ocorrem antes do nascimento adequam a gestante aos protocolos assistenciais do SUS, para que assim, se evite desfechos adversos perinatais. Ou seja, ao longo do acompanhamento pré-natal ocorre rastreio e identificação de possíveis patologias que possam ocorrer durante a gravidez. Além disso, também é avaliado melhor o crescimento e desenvolvimento do feto e, dessa forma, prevenindo casos de prematuridade $(11,12,13)$. 
$\mathrm{Na}$ literatura, há relação entre a prematuridade e atendimentos pré-natais, concluindo que a qualidade do acompanhamento de puérperas, que tiveram recém nascidos prematuros, foi inferior às gestantes que tiveram filho a termo (14). Tal padrão é análogo aos resultados obtidos desse estudo e com o protocolo atual da Organização Mundial da Saúde, os quais indicam que um número maior de consultas irá resultar em um melhor desempenho no acompanhamento gestacional diminuindo, assim, os casos de nascimentos pré-termo. Entretanto, isso diverge das atuais indicações do Ministério da Saúde, que é mais flexível quanto ao número mínimo de atendimentos a gestante.

A prematuridade representa $47 \%$ dos casos de mortalidade infantil, ou seja, é uma patologia que resulta em quase metade dos óbitos na infância. Diante disso, ela também é a maior causa de internação em UTI neonatal e, por consequência, é a maior responsável por óbitos neste período (15). Visto a associação desse desfecho adverso que acarreta em internação na UTI neonatal, constata-se fundamentação baseada na literatura, a qual revela que em mais de $50 \%$ dos casos de bebês prematuros existe algum problema de saúde (15). Por outro lado, as UTI neonatais são muito dispendiosas ao governo e, por isso, o benefício do acompanhamento regular do pré-natal afim de impedir esse desfecho não abrange apenas a saúde maternofetal, mas também, a administração governamental (16).

De acordo com informações já apresentadas, constatou-se uma significativa redução em relação à prematuridade, à medida que ocorre o aumento o número de consultas da gestante. Em função disso, as diretrizes da atenção primária da OMS recomendam 8 ou mais consultas de pré-natal (4), para assim que se configure como fator protetor à prematuridade frente a premissa do Ministério da Saúde, que pede, no mínimo 6 (3). Portanto, recomendações da OMS são mais adequadas, por reduzirem em mais de $20 \%$ os casos desse desfecho em comparação as do Ministério da Saúde

Tendo em vista isso, foi avaliado, também, os níveis de prematuridade (17), divididos em prematuro moderado (maior ou igual a 32 semanas e menor que 37), muito prematuro (entre 28 e 32 semanas) ou extremamente prematuro (menor que 28 semanas). Entretanto, a classificação do desfecho não se mostrou significativa, o que indica que a prematuridade aumentou proporcionalmente nas classificações analisadas em gestantes que realizaram 5 consultas ou menos. No que tange a prematuridade moderada, compreendese que estas pacientes estavam em desacordo com ambos os protocolos, uma vez que com mais de 32 semanas apresentariam um número superior a 6 consultas. Logo, vê-se que é um problema de ordem social, pois irregularidade na frequência do pré-natal é influenciado pelo perfil socioeconômico da mãe (18).

Por outro lado, em relação aos casos de RN muito prematuro ou prematuro extremo, a verificação desses casos não indica um problema na assiduidade da paciente nas consultas, pois em casos de extrema prematuridade não conseguem realizar as 6 consultas requisitadas na atenção primária. Esses casos podem ser resultados de falhas na rede básica, ou até mesmo uma incoerência nos protocolos, uma vez que não transcorreu a identificação e classificação de risco, afim do acompanhamento adequado da gestante. Dentro 
do limitado tempo que é disposto a isso, no início da gravidez, deve ocorrer o rastreamento de fatores de risco responsáveis pelos casos de muito prematuro e prematuridade extrema (19).

Assim, dos resultados obtidos também foi revelado que a prematuridade foi significativa, porém não afetou o BPN e a UTI neonatal. Isso ocorre pois, em casos de prematuridade moderada, o recém-nascido pode apresentar uma massa mais próxima da adequada e não se observa, portanto, razão de chance de UTI neonatal, $(20,21)$ o que se mostra visível também pela insignificância da IG.

Além disso, apresentou-se que conforme aumentava o número de consultas das gestantes, caía o número de casos de internação UTI neonatal. Contudo, ao se observar a razão de chance, esse resultado não se apresentou significativo. Apesar de não haver aumento significativo em uma única classificação de prematuridade, entretanto, de forma constante em todas. Os casos de prematuridade na faixa tardia (maiores ou iguais a 32 semanas e menores que 37 semanas) contribuiu para o não aumentou na internação em UTI neonatal. Tal fato acontece uma vez que o grupo não configura, necessariamente, complicações ao RN que exijam UTI neonatal, por ter risco de complicações algumas vezes menores que bebês pré-termo (22). Análogo a isso, vê-se também que bebês com menor peso ao nascer estão relacionados de modo menos frequente a intercorrências no parto como distorcia de ombro e outras complicações relacionados à macrossomia e o pós-datismo (23)

O Baixo Peso ao Nascer (BPN) foi significativo entre as doenças do recém-nascido, mas não no cálculo da razão de chance mostrando insignificância nos 3 grupos estudados. Esse resultado diferiu da literatura que apresenta o maior número de consultas como fator de proteção em gestantes que realizaram 5 consultas ou menos, por existir maior dificuldade em realizar o acompanhamento nutricional materno, para adequação do peso do feto com a idade gestacional (7). Por outro lado, o BPN é uma patologia muito relacionada ao tabagismo, por provocar alterações nutricionais no embrião (24), e isso foi uma variável limitante do estudo apresentado (7). Contudo há na literatura outros artigos afirmando que é inequívoco a relação BPN com o número de consultas, mas que existe falta de ensaios clínicos randomizados para o esclarecimento dessa relação (20).

Portanto, em relação ao exposto, pode ser observado que a maioria das gestantes já fazem 8 ou mais consultas, e que elas obtiveram $94 \%$ menos chance de prematuridade em relação às que fizeram 5 consultas ou menos. Não obstante, pacientes que seguiram as recomendações do Ministério da Saúde também apresentaram redução nos casos de prematuridade em $74 \%$, contudo, não foi tão significativo quanto às que adotaram a OMS como padrão. Não houve relevância nos demais desfechos avaliados. Assim, compreende -se a importância do adequado controle pré-natal e da assiduidade da paciente ao longo da gestação, possibilitando a detecção e o controle de patologias e fatores de risco propensores de prematuridade. Isso junto com o acompanhamento pré-natal de no mínimo 8 consultas, previne a prematuridade e, 
consequentemente, reduz gastos públicos como, por exemplo, a economia em UTI neonatal relacionadas a esse desfecho.

\section{CONSIDERAÇÕES FINAIS}

Ao analisar o atendimento pré-natal de baixo risco nas Unidades Básicas de Saúde, ficou evidente que o acompanhamento integral e com um número adequado de consultas é de suma importância para um bom decorrer da gestação e desfechos perinatais satisfatórios. Pois, há uma relação direta com a prematuridade e podendo estar relacionado a outros desfechos.

Conforme os resultados obtidos, a realização de 6 ou 7 consultas de pré-natal reduz $74 \%$ as chances de prematuridade, enquanto a realização de 8 ou mais consultas diminuiu em 94\%. Com isso é notório que as recomendações da Organização Mundial da Saúde são mais adequadas em prevenir esse desfecho frente ao atual protocolo do Ministério da Saúde que recomenda no mínimo 6 consultas. Apesar do Brasil possuir regiões com padrões econômicos distintos, para o governo é mais rentável investir na atenção primária prevenindo essa patogenia, do que depois investir no tratamento. Ou seja, para o governo e para a clínica materno-fetal é benéfico seguir o padrão de no mínimo 8 consultas pré-natal.

Além disso como prevenção da prematuridade, o governo deve dar maior atenção nas primeiras consultas e nos protocolos de admissão afim de prevenir a prematuridade extrema. E por outro lado cobrar da gestante comprometimento e assiduidade nas consultas para que consiga atingir o recomendável. Desta forma prevenindo esse desfecho da gestação.

Não há conflitos de interesses. O projeto não recebeu financiamento. 


\section{REFERÊNCIAS}

1. Nunes JT, Gomes KRO, Rodrigues MTP, Mascarenhas MDM. Qualidade da assistência pré-natal no Brasil: revisão de artigos publicados de 2005 a 2015. Cad Saúde Coletiva [Internet]. 2016 Jun [cited 2019 Sep 2];24(2):252-61. Available http://www.scielo.br/scielo.php?script=sci_arttext\&pid=S1414-462X2016000200252\&lng=pt\&tlng=pt

2. Manual Técnico do Pré-Natal e Puerpério Atenção a Gestante e a Puérpera no SUS-SP [Internet]. [cited 2019 Sep 3]. Available from: http://www.saude.sp.gov.br/resources/ses/perfil/gestor/destaques/atencao-a-gestante-e-a-puerpera-no-sussp/manual-tecnico-do-pre-natal-e-puerperio/manual_tecnicoii.pdf

3. Ministério da Saúde [Internet]. [cited 2019 Sep 3]. Available from: http://bvsms.saude.gov.br/bvs/saudelegis/gm/2000/prt0570_01_06_2000_rep.html

4. OMS publica novas orientações sobre pré-natal para reduzir mortes de mães e bebês | ONU Brasil [Internet]. [cited 2019 Sep 2]. Available from: https://nacoesunidas.org/oms-publica-novas-orientacoessobre-pre-natal-para-reduzir-mortes-de-maes-e-bebes/

5. Quadrado Da Rosa C. Fatores associados à não realização de pré-natal em município de grande porte Factors associated with lack of prenatal care in a large municipality. Rev Saúde Pública [Internet]. 2014 [cited 2019 Sep 3];48(6):977-84. Available from: www.scielo.br/rsp

6. Serruya SJ, Cecatti JG, Lago T di G do. O Programa de Humanização no Pré-natal e Nascimento do Ministério da Saúde no Brasil: resultados iniciais. Cad Saude Publica [Internet]. 2004 Oct [cited 2019 Sep 3];20(5):1281-9. Available from: http://www.scielo.br/scielo.php?script=sci_arttext\&pid=S0102$311 \mathrm{X} 2004000500022 \& \operatorname{lng}=\mathrm{pt} \& \operatorname{tlng}=\mathrm{pt}$

7. Universidade Federal de Goiás. Faculdade de Enfermagem. Revista eletrônica de enfermagem : órgão da Faculdade de Enfermagem da Universidade Federal de Goiás. [Internet]. Faculdade de Enfermagem da Universidade Federal de Goiás; 1999 [cited 2019 Oct 16]. Available from: https://www.fen.ufg.br/revista/revista6_3/04_Original.html

8. cuidado prenatal en Brasil E, F Viellas CE, Fernandes Viellas E, Maria Soares Madeira Domingues R, Augusto Bastos Dias M, Granado Nogueira da Gama S, et al. Prenatal Care; Maternal and Child Health; Maternal-Child Health Services. [cited 2019 Dec 17]; Available from: http://dx.doi.org/10.1590/0102$311 \mathrm{X} 00126013$

9. Quadrado Da Rosa C. Fatores associados à não realização de pré-natal em município de grande porte Factors associated with lack of prenatal care in a large municipality. Rev Saúde Pública [Internet]. 2014 [cited 2019 Nov 25];48(6):977-84. Available from: www.scielo.br/rsp

10. Naliato Perez Neto MI, Aparecida De Mattos Segre C. Comparative analysis of gestations and frequency of prematurity and low birth weight among children of adolescent and adult mothers. Vol. 10. 2012. Disponível: https://journal.einstein.br/article/comparative-analysis-of-gestations-and-frequency-ofprematurity-and-low-birth-weight-among-children-of-adolescent-and-adult-mothers/

11. BVS - Ministério da Saúde - Dicas em Saúde [Internet]. [cited 2019 Sep 3]. Available from: http://bvsms.saude.gov.br/bvs/dicas/90prenatal.html 
12. Adauto Martins Soares Filho, Ana Sudária de Lemos Serra, Carmem Lucia de Simoni, Carolina Pereira Lobato, Charleni Inês Scherer, Daisy Maria Coelho de Mendonça, et al. CADERNOS DE ATENÇÃO BÁSICA ATENÇÃO AO PRÉ-NATAL DE BAIXO RISCO [Internet]. $1^{\circ}$. Hêider Aurélio Pinto, editor. Brasília: Ministério da Saúde; 2012 [cited 2019 Oct 16]. 320 p. Available from: http://bvsms.saude.gov.br/bvs/publicacoes/cadernos_atencao_basica_32_prenatal.pdf

13. Machado AKF, Marmitt LP, Cesar JA, Machado AKF, Marmitt LP, Cesar JA. Late preterm birth in the far south of Brazil: a population based study. Rev Bras Saúde Matern Infant [Internet]. 2016 Jun [cited 2019 Oct 16];16(2):113-20. Available from: http://www.scielo.br/scielo.php?script=sci_arttext\&pid=S1519-38292016000200113\&lng=en\&tlng=en

14. Melo EC, Rosseto De Oliveira R, Aidar T, Mathias F. ArtIgO OrIgInAl. [cited 2019 Sep 3];540. Available from: www.ee.usp.br/reeusp

15. Mora Moníi Fonsooa Oarmen Groeinda Silvan Seoehi L. 8JEITaS DE MUDAR OMUNDO Ouidados eom O h h\&amp; pr m tur [Internet]. 2009 [cited 2019 Sep 3]. Available from: http://bvsms.saude.gov.br/bvs/publicacoes/cuidados_bebe_prematuro_3ed.pdf

16. Mwamakamba LW, Zucchi P, Mwamakamba LW, Zucchi P. Cost estimate of hospital stays for premature newborns of adolescent mothers in a Brazilian public hospital. Einstein (São Paulo) [Internet]. 2014 Jun [cited 2019 Oct 16];12(2):223-9. Available from: http://www.scielo.br/scielo.php?script=sci_arttext\&pid=S1679-45082014000200223\&lng=en\&tlng=en

17. Formiga CKMR, Vieira MEB, Linhares MBM. DEVELOPMENTAL ASSESSMENT OF INFANTS BORN PRETERM: COMPARISON BETWEEN THE CHRONOLOGICAL AND CORRECTED AGES. J Hum Growth Dev [Internet]. 2015 Oct 20 [cited 2019 Sep 3];25(2):230. Available from: http://www.revistas.usp.br/jhgd/article/view/103020

18. Rosa CQ da, Silveira DS da, Costa JSD da, Rosa CQ da, Silveira DS da, Costa JSD da. Factors associated with lack of prenatal care in a large municipality. Rev Saude Publica [Internet]. 2014 Dec [cited 2019 Oct 16];48(6):977-84. Available from: http://www.scielo.br/scielo.php?script=sci_arttext\&pid=S0034-89102014000600977\&lng=en\&tlng=en

19. Ramos HÂ de C, Cuman RKN. Fatores de risco para prematuridade: pesquisa documental. Esc Anna Nery [Internet]. 2009 Jun [cited 2019 Oct 16];13(2):297-304. Available from: http://www.scielo.br/scielo.php?script=sci_arttext\&pid=S1414-81452009000200009\&lng=pt\&tlng=pt

20. Kilsztajn S, Rossbach A, Carmo MSN do, Sugahara GTL. Assistência pré-natal, baixo peso e prematuridade no Estado de São Paulo, 2000. Rev Saude Publica [Internet]. 2003 Jun [cited 2019 Sep 3];37(3):303-10. Available from: http://www.scielo.br/scielo.php?script=sci_arttext\&pid=S003489102003000300007\&lng=pt\&tlng=pt

21. Araújo BF de, Tanaka AC d' A, Madi JM, Zatti H. Estudo da mortalidade de recém-nascidos internados na UTI neonatal do Hospital Geral de Caxias do Sul, Rio Grande do Sul. Rev Bras Saúde Matern Infant [Internet]. 2005 Dec [cited 2019 Oct 16];5(4):463-9. Available from: http://www.scielo.br/scielo.php?script=sci_arttext\&pid=S1519-38292005000400010\&lng=pt\&tlng=pt

22. Machado LC, Passini Júnior R, Rodrigues Machado Rosa I, Machado LC, Passini Júnior R, Rosa IRM. Late prematurity: a systematic review. J Pediatr (Rio J) [Internet]. 2014 May [cited 2019 Oct 16];90(3):221-31. Available from: https://linkinghub.elsevier.com/retrieve/pii/S0021755714000175 
23. Robert L. Stavis. Recém-nascido grande para a idade gestacional (GIG) - Pediatria - Manuais MSD edição para profissionais [Internet]. [cited 2019 Oct 16]. Available from: https://www.msdmanuals.com/ptbr/profissional/pediatria/problemas-perinatais/recém-nascido-grande-para-a-idade-gestacional-gig

24. Siqueira AAF de, Santos JLF, Silva JF da. Relação entre estado nutricional da gestante, fumo durante a gravidez, crescimento fetal e no primeiro ano de vida. Rev Saude Publica [Internet]. 1986 Dec [cited 2019

Oct

16];20(6):421-34.

Available from: http://www.scielo.br/scielo.php?script=sci_arttext\&pid=S0034-89101986000600004\&lng=pt\&tlng=pt 